MATHEMATICS OF COMPUTATION

Volume 71, Number 240, Pages 1405-1419

S 0025-5718(01)01369-2

Article electronically published on November 20, 2001

\title{
POSITIVITY PRESERVING FINITE ELEMENT APPROXIMATION
}

\author{
RICARDO H. NOCHETTO AND LARS B. WAHLBIN
}

\begin{abstract}
We consider finite element operators defined on "rough" functions in a bounded polyhedron $\Omega$ in $\mathbb{R}^{N}$. Insisting on preserving positivity in the approximations, we discover an intriguing and basic difference between approximating functions which vanish on the boundary of $\Omega$ and approximating general functions which do not. We give impossibility results for approximation of general functions to more than first order accuracy at extreme points of $\Omega$. We also give impossibility results about invariance of positive operators on finite element functions. This is in striking contrast to the well-studied case without positivity.
\end{abstract}

\section{INTRODUCTION}

Let $\Omega$ be a bounded polyhedral domain in $\mathbb{R}^{N}, N \geq 2$, with simplicial edgeto-edge partitions $\mathfrak{T}$ with local mesh size $h_{T}=\operatorname{diam} T$ for $T \in \mathfrak{T}$. For ease of presentation in this introduction, we assume that the partitions $\mathfrak{T}$ are shape-regular and also quasi-uniform; i.e., there exist two constants $C_{1}$ and $C_{2}$ such that

For any simplex $T \in \mathfrak{T}$, the ratio of the radius of the smallest ball containing $T$ to that of the largest ball contained in $T$ is bounded above by $C_{1}$;

and

$$
\frac{\max _{T \in \mathfrak{T}} h_{T}}{\min _{T \in \mathfrak{T}} h_{T}} \leq C_{2} .
$$

With standard (slight) abuse of notation, we then simply write $h$ for the mesh size $\max _{T \in \mathfrak{T}} h_{T}$.

Let $S_{k}(\Omega ; h)$ be the space of continuous Lagrange finite elements of total polynomial degree $\leq k$, with $k \geq 1$. We start with a brief review of some aspects of approximation theory into these spaces when positivity is not required. Letting $\mathfrak{L}_{k}\left(=\mathfrak{L}_{k, h}\right)$ be the standard pointwise interpolation operator at the nodes (the principal lattice points in each element), we then have that $\mathfrak{L}_{k}$ is up to $(k+1)$-th order accurate in $L_{p}(\Omega)$; i.e.,

$$
\left\|u-\mathfrak{L}_{k}(u)\right\|_{L_{p}(\Omega)} \leq C h^{s}\|u\|_{W_{p}^{s}(\Omega)}, \quad \text { for integers } s \leq k+1,
$$

Received by the editor November 5, 1999 and, in revised form, November 21, 2000.

2000 Mathematics Subject Classification. Primary 41A25, 41A36, 65D05, 65N15, 65N30.

Key words and phrases. Positive operators, finite elements, extreme points, second order accuracy. 
provided also that $N / s<p \leq \infty$ (see [1, Section 4.4] and 4, Section 16]). The condition $p s>N$ guarantees, via Sobolev's inequality, that functions in $W_{p}^{s}(\Omega)$ possess point values and hence that $\mathfrak{L}_{k}(u)$ is well defined.

The needs of numerical analysis of finite element methods for partial differential equations has led to the construction of approximation operators defined on functions without point values, such as those in $W_{p}^{s}(\Omega)$ for $p s \leq N$ (the case $p=1, s=N$ is exceptional in that then point values are defined). As typical examples of such needs, in the context of second order elliptic problems, we mention the following: the a priori error analysis in the energy norm $W_{2}^{1}(\Omega)$ of singular functions $u \in W_{2}^{s}(\Omega)$ with $1<s<2$ [1, Section 5.8], 4, Section 18]; the basic residual-based a posteriori error analysis in $W_{2}^{1}(\Omega)$, which requires approximation of functions in $W_{2}^{1}(\Omega)$ [12, Section 1.2]; the local construction of Fortin's operators for mixed finite element methods, which deals with local approximation of functions in $H(\operatorname{div} ; \Omega)$ [2, Section III.3]; and the analysis of pointwise errors for function values (or gradients), which is often based on approximation of functions in $W_{1}^{2}(\Omega)$ (or $W_{1}^{1}(\Omega)$ ) such as regularized Green's functions [1, Section 7.2].

Approximation operators as above necessarily involve averaging; we refer to Clément [5, Hilbert [6], Scott and Zhang [10, and Strang [11] for details of different such constructions and analyses of their properties. For approximation on a polyhedral domain, as is our present case, the Scott-Zhang operator $\mathfrak{S}_{k}\left(=\mathfrak{S}_{k, h}\right)$ into the Lagrange finite elements is of particular interest for later comparison when we also demand positivity. We shall not actually define this operator, since it is somewhat lengthy to do so and not essential for our purposes, but we record here some of its properties [10]:

$\mathfrak{S}_{k}$ satisfies (1.3) for all $1 \leq p \leq \infty$ provided $s \geq 1$ (which guarantees that functions in $W_{p}^{s}(\Omega)$ have well-defined traces on $\partial \Omega$ ); also, if $u=0$ on $\partial \Omega$, then $\mathfrak{S}_{k}(u)=0$ on $\partial \Omega$;

and

$$
\begin{aligned}
& \mathfrak{S}_{k} \text { reproduces all the Lagrange finite element functions of degree } \\
& \leq k ; \text { i.e., if } u \in S_{k}(\Omega ; h) \text {, then } \mathfrak{S}_{k}(u)=u \text {. }
\end{aligned}
$$

For the analysis of finite element methods for variational inequalities [3] it is natural and sometimes instrumental to have approximation operators $\mathfrak{I}\left(=\mathfrak{I}_{h}\right)$ which are also positive:

$$
u \geq 0 \text { implies } \Im(u) \geq 0 .
$$

Of course, if $u$ does have point values, then the piecewise linear pointwise interpolation operator $\mathfrak{L}_{1}$ is such a positive operator (but the general Lagrange interpolation operator $\mathfrak{L}_{k}$ is not for $k \geq 2$ ). None of the averaging approximation operators of [5], 6], [10, [11] is positive. Moreover, simple truncation at the nodal values of $\Im(u)$ would enforce positivity for $k=1$ (not for $k \geq 2$ ), but at the expense of linearity, a crucial and desirable property of $\mathfrak{I}$. For rough functions without point values, a linear and positive operator $\mathfrak{I}: L_{p}(\Omega) \rightarrow \stackrel{\circ}{S}_{1}(\Omega ; h)$ has been introduced in Chen and Nochetto [3] with values into the spaces $\stackrel{\circ}{1}_{1}(\Omega ; h)$ of piecewise linear finite elements which vanish on $\partial \Omega$.

For the purpose of discussing our major impossibility result, Theorem 1 below, it will be convenient to have a brief description of this operator. Thus let $B(x, r)$ denote the open ball of center $x$ and radius $r$, and note that the nodes $x_{i}$ of $\mathfrak{T}$ are 
the vertices of the simplices in the piecewise linear situation. For nodes $x_{i}$ which are interior to $\Omega$, we define the nodal value of $\mathfrak{I}$ as the local mean-value

$$
\mathfrak{I}\left(u ; x_{i}\right):=\frac{1}{\operatorname{meas}\left(B_{i}\right)} \int_{B_{i}} u,
$$

where $B_{i}:=B\left(x_{i}, C_{3} h\left(x_{i}\right)\right)$ with $C_{3}$ so small that it is contained in the star associated with $x_{i}$ (i.e., the union of closed elements containing $x_{i}$ ). At nodes $x_{i}$ on $\partial \Omega$, we set $\Im\left(u ; x_{i}\right):=0$; thus $\mathfrak{I}(u) \in \stackrel{\leftrightarrow}{S}_{1}(\Omega ; h)$ for all $u \in L_{p}(\Omega)$.

It was shown in [3] that $\mathfrak{I}$ satisfies (1.3) for $u \in W_{p}^{s}(\Omega) \cap \stackrel{\circ}{W}_{p}^{1}(\Omega), s=1,2$, and all $p \geq 1$. Hence, $\mathfrak{I}$ is second order accurate in $L_{p}(\Omega)$ for functions on $W_{p}^{2}(\Omega)$ which vanish on $\partial \Omega$. The two essential ideas involved in the proof are:

- The simple averaging in (1.7) implies that all nodal values are nonnegative for nonnegative functions, whence $\mathfrak{I}$ satisfies (1.6);

- Due to the symmetry of the interior balls $B_{i}$ with respect to $x_{i}$, any locally affine function $u$ would be reproduced locally, i.e., $\mathfrak{I}\left(u ; x_{i}\right)=u\left(x_{i}\right)$.

Then standard approximation theory for finite elements based on a circle of ideas involving local polynomial invariance (in the present case the local affine functions), in conjunction with some extra arguments from [10] to handle vanishing boundary values, gives (1.3) for $s=1$ and 2. This circle of ideas has been pervasive in the finite element literature for more than thirty years now; see, e.g., Brenner and Scott [1], Brezzi and Fortin [2] and Ciarlet [4].

Let us now remark that it is not possible to ask for more than second order accuracy in positive approximation operators, if they are also required to be linear operators, no matter how smooth the functions to be approximated are. This is a classical result of Korovkin [7, given by him in the context of polynomial approximation with increasing degree on a fixed domain. His ideas translate to our finite element context, and thus one cannot achieve (1.3) with $s>2$ for positive operators which are also linear, irrespective of the polynomial degree.

We now turn to our basic problem in this paper:

Is it possible to construct bounded and positive (linear) operators into $S_{1}(\Omega ; h)$ also for functions which do not necessarily vanish on $\partial \Omega$ ?

As already mentioned, if $p s>N$ so that the function $u$ has point values, we may simply use the piecewise linear Lagrange operator $\mathfrak{L}_{1}$. In order to motivate our impossibility result, we next consider two examples.

Example 1.1 (First order approximation). Here we ask for positive operators $\mathfrak{I}$ : $L_{p}(\Omega) \rightarrow S_{1}(\Omega ; h)$, defined for functions without point values, which satisfy (1.3) with $s=1$ for all $p \geq 1$. We construct $\mathfrak{I}$ as follows: For interior nodes, let the nodal values be as in (1.7). At boundary nodes $x_{i}$, let $D_{i}:=B\left(x_{i}, C_{3} h\left(x_{i}\right)\right) \cap \Omega$ and

$$
\mathfrak{I}\left(u ; x_{i}\right):=\frac{1}{\operatorname{meas}\left(D_{i}\right)} \int_{D_{i}} u .
$$

Since constant functions are locally reproduced, using standard techniques it is easy to verify (1.3) for $s=1$ and all $p \geq 1$. However, at the boundary the domains $D_{i}$ are not symmetric with respect to the nodes $x_{i}$, affine functions are not reproduced locally, and (1.3) is not satisfied for $s=2$.

We next attempt second order approximation for a simple domain in two dimensions. 
Example 1.2 (Second order approximation?). Let $\Omega$ be an L-shaped domain in the plane and let $\mathfrak{I}: W_{p}^{1}(\Omega) \rightarrow S_{1}(\Omega ; h)$ be defined as follows. For interior nodes, we again use (1.7). As for boundary nodes $x_{i}$, we distinguish between three kind of nodes: the nodes which are interior to a boundary straight segment $\left\{x_{i}^{S}\right\}$; the lone node at the reentrant (nonconvex) corner $x^{R}$; and the five nodes at the convex corners $\left\{x_{i}^{C}\right\}$. For $x_{i}^{S}$, we let $L_{i}:=B\left(x_{i}^{S}, C_{3} h\left(x_{i}^{S}\right)\right) \cap \partial \Omega$ be a straight segment which is symmetrically placed around $x_{i}^{S}$ and in $\partial \Omega$. Then we define the nodal value at $x_{i}^{S}$ as the local line integral mean-value

$$
\Im\left(u ; x_{i}^{S}\right):=\frac{1}{\operatorname{length}\left(L_{i}\right)} \int_{L_{i}} u .
$$

This is well defined since, for $s \geq 1$ and all $p \geq 1$, the functions in $W_{p}^{s}(\Omega)$ have traces on $\partial \Omega$. At $x^{R}$, we may similarly place a small line-segment $L^{R}$ contained in $\bar{\Omega}$ which is symmetric about $x^{R}$ and define the nodal value $\mathfrak{I}\left(u ; x^{R}\right)$ accordingly. Due to the symmetric placement of discs and line-segments, we have preservation of local affine functions and thus, so far, local second order accuracy. However, at the five convex corners $x_{i}^{C}$, this idea breaks down. A similar symmetric line-segment would have to extend outside of $\bar{\Omega}$.

The problematic convex corners in Example 1.2 point to the heart of the matter. For a general polyhedral domain in $\mathbb{R}^{N}$, we have the standard notion of extreme points $e$ : these are points on $\partial \Omega$ such that there is a supporting hyperplane at $e$; i.e., there is an affine function $a_{e}(x)$ satisfying

$$
a_{e}(e)=0 \quad \text { and } \quad a_{e}(x)>0 \quad \text { for all } x \in \bar{\Omega}, x \neq e .
$$

Note that any bounded domain has extreme points.

Now we are ready to give our major impossibility result (in the simplifying context when shape-regularity (1.1) and quasi-uniformity (1.2) are satisfied). With a fixed $p \leq N$, we are seeking linear approximation operators $\mathfrak{I}_{h}$ into $S_{k}(\Omega ; h)$ which are positive and, as a basic minimal requirement, are also first order accurate:

$$
\left\|u-\Im_{h}(u)\right\|_{L_{p}(\Omega)} \leq C h\|u\|_{W_{p}^{1}(\Omega)}, \quad \text { for all } u \in W_{p}^{1}(\Omega) .
$$

Example 1.1 shows that such $\mathfrak{I}_{h}$ do exist. We now add a further demand which involves superlinear (higher than first order) approximation but only for the affine functions and only at an extreme point. With $a_{0}(x)=1, a_{n}(x)=x_{n}$ for $1 \leq n \leq N$, we thus assume that $\mathfrak{I}_{h}$ satisfies at an extreme point $e$, with some $\gamma>0$,

$$
\left|a_{n}(e)-\Im_{h}\left(a_{n} ; e\right)\right| \leq C_{\gamma} h^{1+\gamma}, \quad \text { for } 0 \leq n \leq N .
$$

Theorem 1. Assume (1.1), (1.2), and let $1 \leq p \leq N, N \geq 2, k \geq 1$. There do not exist positive linear operators $\mathfrak{I}_{h}: W_{p}^{1}(\Omega) \rightarrow S_{k}(\Omega ; h)$ which satisfy both (1.9) and (1.10) as $h$ tends to zero.

Note that, without the positivity requirement, the Scott-Zhang operator $\mathfrak{S}$ satisfies (1.9) and (1.10), the latter being satisfied even in the stronger sense that $\mathfrak{S}\left(a_{n}\right)=a_{n}$ for $0 \leq n \leq N$.

We next present a result which complements and gives insight into the impossibility result of Theorem 10 The context now does not involve any finite element spaces but only a single operator $\mathfrak{I}$ on the space $C(\bar{\Omega})$ of continuous functions in $\bar{\Omega}$. 
Theorem 2. Let $\mathfrak{I}: C(\bar{\Omega}) \rightarrow C(\bar{\Omega})$ be a linear, bounded, and positive operator. Let $\mathfrak{I}$ reproduce exactly all affine functions: $\mathfrak{I}\left(a_{n}\right)=a_{n}$ for $0 \leq n \leq N$. Then, at any extreme point e of $\Omega$, we have

$$
\Im(u ; e)=u(e), \quad \text { for all } u \in C(\bar{\Omega}) .
$$

Again, without the positivity requirement, the Scott-Zhang operator on any fixed mesh and for any $k \geq 1$ satisfies the basic assumptions of Theorem 2 but not the conclusion (1.11).

Since second order accuracy in the finite element context is, essentially, equivalent to (locally) preserving affine functions, Theorems 1 and 2 together uncover the amazing and unexpected fact that, for a positive approximation operator $\mathfrak{I}$ to be of second order, one does indeed need point values of functions at extreme points and $\mathfrak{I}$ reduces to point evaluation at those points.

The impossibility result, Theorem 1, and the complementing result, Theorem 2 may be loosely described as results pertaining to (approximately) reproducing affine functions (either locally or globally). In finite element analysis it is often convenient if approximation operators also reduce to the identity operator when applied to functions in the finite element space. This is the case for the ScottZhang operator without the positivity requirement (see (1.5)). For the positive Chen-Nochetto operator, the situation is drastically different as is readily seen by applying it to a piecewise linear basis function. We refer to this new circle of problems as pertaining to (approximately) reproducing piecewise affine functions. We give, in the next two sections, results analogous to the impossibility result Theorem 1 and the complementing result Theorem 2 also for this circle of problems.

We now outline the rest of the paper. It is organized in a way that does not correspond to the order in which results have been discussed so far. It rather reflects a natural grouping of results with respect to the techniques of proofs involved, and it also reflects a natural progression of these techniques. In Section 2 we prove complementing results such as Theorem 2 in the context of reproducing affine, respectively piecewise affine, functions. In Section 3 we derive corresponding impossibility results such as Theorem 10 Finally, in Section 4 we discuss the limitation to second order accuracy imposed by positivity in the finite element context. We have not been able to find this extension of Korovkin's results to piecewise polynomial approximation in the literature, but think it is of value to make it available to the numerical analysis community. The extension is not entirely obvious.

Throughout this paper, $C$ will denote a positive constant, not necessarily the same at each occurrence. It will always be independent of $h$ unless otherwise specified.

\section{Complementing Results in $C(\bar{\Omega})$}

We consider linear positive operators $\mathfrak{I}$ which are bounded in the space of continuous functions and reproduce affine or piecewise linear functions. The complementing result in the introduction, Theorem 2, as well as other companion results will follow from Theorem 3 below. We prove such a basic Theorem 3 in its simplest form and comment on possible extensions in Remark 2.3

Let $C(\bar{\Omega})$ be the space of continuous functions on $\bar{\Omega}$, and let $\stackrel{\circ}{C}(\Omega)$ be the subspace of functions which vanish on $\partial \Omega$. The $\operatorname{support} \operatorname{supp}(u)$ of a function $u$ is the closed 
subset of $\bar{\Omega}$ defined by

$$
\operatorname{supp}(u)=\text { closure of }\{x \in \bar{\Omega}: u(x) \neq 0\} .
$$

Note that the support may coincide with $\bar{\Omega}$ for a function in $\stackrel{\circ}{C}(\Omega)$.

Theorem 3. Let $\hat{\Omega}$ indicate either $\Omega$ or $\bar{\Omega}$ and let $\hat{C}(\hat{\Omega})$ be $\stackrel{\circ}{C}(\Omega)$ or $C(\bar{\Omega})$, accordingly. Let $\mathfrak{I}: \hat{C}(\hat{\Omega}) \rightarrow C(\bar{\Omega})$ be a linear, bounded, and positive operator. Let $x_{0} \in \hat{\Omega}$ and $f_{0}, f_{+} \in \hat{C}(\hat{\Omega})$ satisfy

$$
\begin{gathered}
\Im\left(f_{0} ; x_{0}\right)=f_{0}\left(x_{0}\right)=1, \\
f_{+}(x)>0, \quad \forall x \in \hat{\Omega} \backslash\left\{x_{0}\right\}, \\
\Im\left(f_{+} ; x_{0}\right)=0 .
\end{gathered}
$$

Then $\mathfrak{I}\left(u ; x_{0}\right)=u\left(x_{0}\right)$ for all $u \in \hat{C}(\hat{\Omega})$.

Proof. We first observe that it suffices to demonstrate the assertion for functions which vanish at $x_{0}$. In fact, let $w=u-u\left(x_{0}\right) f_{0} \in \hat{C}(\hat{\Omega})$ which satisfies $w\left(x_{0}\right)=0$ and $\mathfrak{I}\left(w, x_{0}\right)=\mathfrak{I}\left(u ; x_{0}\right)-u\left(x_{0}\right)$ because of (2.1).

We next see by standard density (approximation) arguments that it is enough to consider functions $w$ whose support avoids $x_{0}$ (and avoids $\partial \Omega$ in case of functions which vanish on $\partial \Omega$ ); i.e., $\operatorname{supp}(w)$ is a compact set in the set $\hat{\Omega} \backslash\left\{x_{0}\right\}$. Hypothesis (2.2) yields the existence of a positive number $\alpha$ such that

$$
f_{+}(x) \pm \alpha w(x) \geq 0, \quad \forall x \in \hat{\Omega} .
$$

Using also (2.3), we then have

$$
0=\mathfrak{I}\left(f_{+} ; x_{0}\right)=\mathfrak{I}\left(f_{+} \pm \alpha w ; x_{0}\right) \mp \alpha \mathfrak{I}\left(w ; x_{0}\right),
$$

where the first term on the right is nonnegative regardless of the choice of sign. This implies that $\mathfrak{I}\left(w ; x_{0}\right)=0$ and concludes the proof.

Our first corollary is Theorem 2 of the introduction.

Corollary 1. Let $\mathfrak{I}: C(\bar{\Omega}) \rightarrow C(\bar{\Omega})$ reproduce all affine functions and let $x_{0}$ be an extreme point of $\bar{\Omega}$. Then

$$
\Im\left(u ; x_{0}\right)=u\left(x_{0}\right), \quad \forall u \in C(\bar{\Omega}) .
$$

Proof. Simply take $f_{0}=1$ and $f_{+}=a_{0}$, the affine function of (1.8) associated with $x_{0}$, and apply Theorem 3

Corollary 1 pertains to reproducing affine functions. Our next two applications of Theorem 3 correspond to reproducing piecewise linear functions over $\mathfrak{T}$ or, equivalently, to invariance of $\mathfrak{I}$ in subspaces of $S_{1}(\Omega ; h)$. We recall that if $\left\{\phi_{j}\right\}_{j=1}^{J}$ are the canonical piecewise linear basis functions, i.e., $\phi_{j}\left(x_{i}\right)=\delta_{i j}$ at nodes $x_{i}$ (including boundary nodes), then $S_{1}(\Omega ; h)=\operatorname{span}\left\{\phi_{j}\right\}_{j=1}^{J}$; note that $1 \in S_{1}(\Omega ; h)$.

Corollary 2. Let $\mathfrak{I}: C(\bar{\Omega}) \rightarrow C(\bar{\Omega})$ reproduce the functions of $S_{1}(\Omega ; h)$ and let $x_{i}$, $1 \leq i \leq J$, be any node of the partition $\mathfrak{T}$. Then

$$
\Im\left(u ; x_{i}\right)=u\left(x_{i}\right), \quad \forall u \in C(\bar{\Omega}) .
$$

Proof. Take $f_{0}=\phi_{i}$ and $f_{+}=1-\phi_{i}$, and apply Theorem 3 
Let $\stackrel{\circ}{S}_{1}(\Omega ; h)=\operatorname{span}\left\{\phi_{i}\right\}_{i=1}^{I}$ be the subspace of piecewise linear functions of $S_{1}(\Omega ; h)$ vanishing on $\partial \Omega$. Now letting $\bar{\Omega}_{0}$ denote the union of all closed simplices which do not have any vertex on $\partial \Omega$, we have

$$
1_{0}(x):=\sum_{i=1}^{I} \phi_{i}(x)=1, \quad \forall x \in \bar{\Omega}_{0} .
$$

Corollary 3. Let $\mathfrak{I}: \stackrel{\circ}{C}(\Omega) \rightarrow C(\bar{\Omega})$ reproduce the functions in $\stackrel{\circ}{S}_{1}(\Omega ; h)$ and let $x_{0}$ be an interior node such that the support $\operatorname{supp}\left(\phi_{0}\right)$ of its canonical basis function $\phi_{0}$ does not meet $\partial \Omega$. Then

$$
\Im\left(u ; x_{0}\right)=u\left(x_{0}\right), \quad \forall u \in \stackrel{\circ}{C}(\Omega) .
$$

Proof. Let $f_{0}=\phi_{0}$, and set $\Xi_{0}=\operatorname{supp}\left(\phi_{0}\right)$. Since $\Xi_{0}$ does not intersect $\partial \Omega$, we have by (2.4) that $1_{0}(x)=1$ for all $x \in \Xi_{0}$. Let $f_{+}=1_{0}-\phi_{0}$; then $f_{+}>0$ in $\Omega \backslash\left\{x_{0}\right\}$. Now apply Theorem 3 .

Remark 2.1. Let $\bar{\Omega}=[0,1]$ and $\mathfrak{T}$ be a uniform mesh of size $h=(I+1)^{-1}$. Let $\left\{x_{i}\right\}_{i=1}^{I}$ be the interior vertices and $\left\{\phi_{i}\right\}_{i=1}^{I}$ the corresponding basis functions of $\stackrel{\circ}{S}_{1}(\Omega ; h)$. Define $\mathfrak{I}: \stackrel{\circ}{C}(\Omega) \rightarrow \stackrel{\circ}{S}_{1}(\Omega ; h)$ by

$$
\mathfrak{I}(u)=2 u(h / 2) \phi_{1}+\sum_{i=2}^{I-1} u\left(x_{i}\right) \phi_{i}+2 u(1-h / 2) \phi_{I} .
$$

Since this operator $\mathfrak{I}$ reproduces $\stackrel{\circ}{S}_{1}(\Omega ; h)$, but does not reproduce point values at $x_{1}$ and $x_{I}$, we see that our condition that $\operatorname{supp}\left(\phi_{0}\right)$ avoids $\partial \Omega$ is necessary in Corollary 3

Remark 2.2. Let $h(x)$ be a piecewise linear mesh density function equivalent to the local mesh size of $\mathfrak{T}$. Consider the positive and bounded linear operator $\mathfrak{I}: C(\bar{\Omega}) \rightarrow$ $C(\bar{\Omega})$ given by

$$
\mathfrak{I}(u ; x)=\mathfrak{L}_{1}(u ; x)+u\left(x_{0}\right) h(x)^{m},
$$

where $x_{0} \in \Omega$ and $m \geq 1$ integer. Then $\mathfrak{I}$ approximates affine or piecewise linear functions to any order $m$, but it does not reproduce them. We conclude that the invariance assumptions of Corollaries 13 are necessary to deduce that $\mathfrak{I}\left(u ; x_{0}\right)=$ $u\left(x_{0}\right)$.

Remark 2.3. The function $f_{+}$of Theorem 3 need not be strictly positive in the entire $\hat{\Omega}$ but rather locally. In fact, consider the case $\hat{\Omega}=\Omega$ and assume that for each $y \in \Omega$ there exists a nonnegative function $f_{+}=f_{+}(y, \cdot) \in \dot{C}(\Omega)$ satisfying $\Im\left(f_{+} ; x_{0}\right)=0$ together with $f_{+}>0$ in a closed ball $\bar{B}(y, \rho) \subset \Omega$ of center $y$ and radius $\rho=\rho(y)$; alternatively, for $\hat{\Omega}=\bar{\Omega}$, assume $f_{+} \in C(\bar{\Omega})$, and $f_{+}>0$ in $\bar{B}(y, \rho) \cap \bar{\Omega}$. To show that the assertion of Theorem 3 still holds, one resorts to a finite covering argument of $\operatorname{supp}(w)$ in conjunction with a partition of unity.

\section{ImpossibiLity RESULts in $W_{p}^{1}(\Omega)$}

In this section we prove Theorem 1 and other impossibility results for $N \geq 2$ and $1 \leq p \leq N$. Their proofs hinge upon constructing suitable functions $v$ in $W_{p}^{1}(\Omega)$ and associated barriers $\phi_{0}$. As a motivation, we give first $v$ and $\phi_{0}$ for a simple and, we hope, illuminating situation. 
Let $F(\Omega) \subset C(\bar{\Omega})$ be a finite-dimensional space of functions containing the affine functions. Let $\mathfrak{I}: W_{p}^{1}(\Omega) \rightarrow F(\Omega)$ be a linear, positive and bounded operator, which reproduces all affine functions. Let $x_{0}=0$ be an extreme point of $\Omega$, and let $v=v_{\varepsilon}$ be given in polar coordinates by $v(r)=\left|\frac{\log \varepsilon}{\log r}\right|$ if $r \leq \varepsilon$ and 1 otherwise. A calculation shows that $v \in W_{p}^{1}(\Omega)$ for all $p \leq N$ and that $\lim _{\varepsilon \rightarrow 0} v=1$ in $W_{p}^{1}(\Omega)$. Since all norms are equivalent in $F(\Omega)$, we deduce that $|\Im(u ; 0)| \leq C_{F}\|u\|_{W_{p}^{1}(\Omega)}$ and that

$$
\Im(v ; 0) \rightarrow \Im(1 ; 0)=1, \quad \text { as } \varepsilon \rightarrow 0 .
$$

Since $\Omega$ is polyhedral and $x_{0}$ is an extreme point, there exists a constant $K>0$ such that the affine function $a$ of (1.8) satisfies

$$
a(x) \geq K\left|x-x_{0}\right|, \quad \forall x \in \bar{\Omega} .
$$

We now set $\phi_{0}(x)=\varepsilon+\beta_{\varepsilon} a(x)$ and realize that $0 \leq v \leq \phi_{0}$ in $\Omega$ provided $\beta_{\varepsilon}$ is sufficiently large. Since $\phi_{0}$ is affine, we arrive at the contradiction

$$
0 \leq \mathfrak{I}(v ; 0) \leq \mathfrak{I}\left(\phi_{0} ; 0\right)=\phi_{0}(0)=\varepsilon .
$$

Consequently, there is no such operator $\mathfrak{I}$.

It is our intention to exploit such an idea for operators $\mathfrak{I}$ which are just almostinvariant on the affine, or piecewise linear, functions (see (1.10) and (3.6) below). We give a general construction in Theorem 4 and we then apply it to specific cases in Corollaries 46 .

In contrast to the introduction, we now consider quite general finite element spaces $S(\Omega ; h)$ over general meshes $\mathfrak{T}$ with some additional properties. We first assume that $S(\Omega ; h)$ satisfy the local inverse assumption

$$
\|u\|_{L_{\infty}(\Omega)} \leq C\left\|h^{-N / p} u\right\|_{L_{p}(\Omega)}, \quad \forall u \in S(\Omega ; h) .
$$

Note that finite element spaces over shape-regular triangulations satisfy (3.2).

Let $x_{0} \in \bar{\Omega}$ be an auxiliary point to be chosen later. We assume that for each $\delta>0$ there exist a function $v \in W_{p}^{1}(\Omega)$ and a barrier function $\phi_{0} \in W_{p}^{1}(\Omega) \cap C(\bar{\Omega})$ such that

$$
\begin{gathered}
\left\|h^{1-N / p} \nabla(v-1)\right\|_{L_{p}(\Omega)}+\left\|h^{-N / p}(v-1)\right\|_{L_{p}(\Omega)} \leq \delta, \\
0 \leq v(x) \leq \phi_{0}(x) \quad \forall x \in \Omega, \quad \text { and } \quad \phi_{0}\left(x_{0}\right)<\frac{1}{2} .
\end{gathered}
$$

Let $h_{0}$ denote the mesh size at $x=x_{0}$.

Theorem 4. There exists no linear, bounded, and positive operator $\mathfrak{I}: W_{p}^{1}(\Omega) \rightarrow$ $S(\Omega ; h)$ which satisfies, for some $\gamma>0$,

$$
\begin{gathered}
\left\|h^{-N / p}(u-\mathfrak{I}(u))\right\|_{L_{p}(\Omega)} \leq C\left\|h^{1-N / p}(|u|+|\nabla u|)\right\|_{L_{p}(\Omega)} \quad \forall u \in W_{p}^{1}(\Omega), \\
\left|1-\mathfrak{I}\left(1 ; x_{0}\right)\right|+\left|\phi_{0}\left(x_{0}\right)-\mathfrak{I}\left(\phi_{0} ; x_{0}\right)\right| \leq C_{\delta} h_{0}^{\gamma},
\end{gathered}
$$

Note that (3.5) and (3.6) state that $\mathfrak{I}$ is locally of first order in $L_{p}(\Omega)$ and that $\mathfrak{I}$ approximates both 1 and $\phi_{0}$ at $x_{0}$, respectively. If the underlying meshes $\mathfrak{T}$ are quasi-uniform, then (3.5) reduces to the more standard condition (1.9).

Proof. Taking $u=v-1$ and applying (3.2) together with (3.5) and (3.3), we obtain

$$
\begin{aligned}
\left|\Im\left(u ; x_{0}\right)\right| & \leq C\left\|h^{-N / p} \mathfrak{I}(u)\right\|_{L_{p}(\Omega)} \\
& \left.\leq C \| h^{-N / p}(\Im)-u\right)\left\|_{L_{p}(\Omega)}+C\right\| h^{-N / p} u \|_{L_{p}(\Omega)} \\
& \leq C\left\|h^{1-N / p}(|u|+|\nabla u|)\right\|_{L_{p}(\Omega)}+C\left\|h^{-N / p} u\right\|_{L_{p}(\Omega)} \leq C \delta .
\end{aligned}
$$


We now choose $\delta$ and then $h_{0}$ sufficiently small and use (3.6) to deduce that

$$
\left|\mathfrak{I}\left(v ; x_{0}\right)-1\right| \leq\left|\mathfrak{I}\left(v-1 ; x_{0}\right)\right|+\left|\mathfrak{I}\left(1 ; x_{0}\right)-1\right| \leq C \delta+C_{\delta} h_{0}^{\gamma}<\frac{1}{4} .
$$

On the other hand, in view of (3.4) and (3.6), for $h_{0}$ perhaps even smaller we arrive at

$$
0 \leq \mathfrak{I}\left(v ; x_{0}\right) \leq \mathfrak{I}\left(\phi_{0} ; x_{0}\right) \leq\left|\mathfrak{I}\left(\phi_{0} ; x_{0}\right)-\phi_{0}\left(x_{0}\right)\right|+\phi_{0}\left(x_{0}\right) \leq C_{\delta} h_{0}^{\gamma}+\frac{1}{2}<\frac{3}{4} .
$$

This is a contradiction which proves the assertion.

Remark 3.1. As is easily seen from the proof, the assumption (3.6) can be replaced by the weaker condition

$$
\left|1-\mathfrak{I}\left(1 ; x_{0}\right)\right|+\left|\phi_{0}\left(x_{0}\right)-\mathfrak{I}\left(\phi_{0} ; x_{0}\right)\right|=o(1) .
$$

Remark 3.2. If the function 1 in (3.3) and (3.6) is replaced by the function $1_{0}$ of (2.4), then Theorem 4 extends to the case $\mathfrak{I}: \dot{W}_{p}^{1}(\Omega) \rightarrow S(\Omega ; h)$.

We now give three applications of Theorem 4. We start with the simplest case $p<N$, which reveals the main idea.

Corollary 4. Let $x_{0}$ be an extreme point of $\Omega$. There is no linear, bounded, and positive operator $\mathfrak{I}: W_{p}^{1}(\Omega) \rightarrow S(\Omega ; h)$ with $1 \leq p<N$ which is first order accurate in $L_{p}(\Omega)$, i.e., satisfies (3.5), and is superlinear in the affine functions $a_{n}$ at $x=x_{0}$, namely,

$$
\left|a_{n}\left(x_{0}\right)-\Im\left(a_{n} ; x_{0}\right)\right|=o\left(h_{0}\right), \quad 0 \leq n \leq N .
$$

Proof. Let $v$ be radially symmetric and given in polar coordinates by $v(x)=\rho(r)$ with $r=\left|x-x_{0}\right|$ and

$$
\rho(r):= \begin{cases}\frac{r}{\varepsilon}, & r \leq \varepsilon \\ 1, & r>\varepsilon\end{cases}
$$

A simple calculation yields

$$
\varepsilon\|\nabla(v-1)\|_{L_{p}(\Omega)}+\|v-1\|_{L_{p}(\Omega)} \leq C \varepsilon^{N / p},
$$

which implies (3.3) provided $\varepsilon=h_{0}\left(\frac{\delta}{C}\right)^{p /(N-p)}$; thus $h_{0}=C_{\delta} \varepsilon$. We have

$$
0 \leq \rho(r) \leq \frac{r}{\varepsilon}=: \varphi(r), \quad \forall r \geq 0 .
$$

Let $a$ be the affine function of (3.1) and let the barrier function $\phi_{0}$ be given by

$$
\phi_{0}(x):=\varphi\left(\frac{a(x)}{K}\right)=\frac{a(x)}{\varepsilon K} .
$$

We observe that $\phi_{0}$ satisfies both conditions in (3.4), the latter with $\phi_{0}\left(x_{0}\right)=0$. To apply Theorem 4 it remains to verify (3.7) for $\phi_{0}$. In view of (3.8), we have

$$
\left|\Im\left(\phi_{0} ; x_{0}\right)\right|=\frac{1}{K \varepsilon}\left|\Im\left(a ; x_{0}\right)\right| \leq \frac{h_{0}}{K \varepsilon} o(1)=o(1),
$$

which implies (3.7). We finally apply Theorem 4 to conclude the proof.

The second consequence of Theorem 4 is similar to Corollary 4 but with $p=N$. We observe that for this critical case we need a function $\rho$ different from (3.9) since the ensuing function $v$ does not satisfy (3.3) any longer. The rather tricky but elementary proof proceeds along the same lines as that of Corollary 4 , but requires a property slightly stronger than (3.8).

Corollary 5. Let (3.8) be replaced by $\left|a_{n}\left(x_{0}\right)-\mathfrak{I}\left(a_{n} ; x_{0}\right)\right| \leq C_{\gamma} h_{0}^{1+\gamma}$ for some $\gamma>0$. Then the assertion of Corollary 4 is also valid for $p=N$. 
Proof. Let $\varepsilon, L$ be two parameters to be chosen later, the former small and the latter large. Let $\rho$ be given by

$$
\rho(r):= \begin{cases}\left|\frac{\log \varepsilon}{\log r}\right|^{L}, & r \leq \varepsilon, \\ 1, & r>\varepsilon,\end{cases}
$$

and set $v(x):=\rho\left(\left|x-x_{0}\right|\right)$. We split the rest of the proof into several steps.

1. We first note that an elementary calculation yields

$$
\begin{gathered}
\|v-1\|_{L_{N}(\Omega)} \leq N^{-1 / N} \varepsilon \leq \varepsilon \\
\|\nabla(v-1)\|_{L_{N}(\Omega)}=\frac{L \omega_{0}^{1 / N}}{(N(L+1)-1)^{1 / N}|\log \varepsilon|^{1-1 / N}} \leq C \frac{L^{1-1 / N}}{|\log \varepsilon|^{1-1 / N}},
\end{gathered}
$$

where $\omega_{0}<\pi$ is the interior angle of $\Omega$ at $x=x_{0}$ and $C$ depends only on $N$. This shows that (3.3) holds for $\varepsilon \leq h_{0}$ sufficiently small since $L$ will be chosen independently of $\varepsilon$ (see (3.10) below).

2. We now construct the barrier function $\phi_{0}$. We have for $r \leq \varepsilon$

$$
\rho^{\prime}(r)=\frac{L|\log \varepsilon|^{L}}{r|\log r|^{L+1}}, \quad \rho^{\prime \prime}(r)=\frac{L|\log \varepsilon|^{L}}{r^{2}|\log r|^{L+2}}(L+1-|\log r|),
$$

whence $\rho^{\prime \prime}(r)<0$ if $r<e^{-(L+1)}$. Therefore, for $\varepsilon$ small, $\rho$ is concave and

$$
0 \leq \rho(r) \leq \rho\left(r_{1}\right)+\rho^{\prime}\left(r_{1}\right)\left(r-r_{1}\right)=: \varphi(r)
$$

for $0<r_{1}<\varepsilon$. In view of (3.1), we set

$$
\phi_{0}(x):=\varphi\left(\frac{a(x)}{K}\right) .
$$

3. With $h_{0}$ sufficiently small, let $\varepsilon, r_{1}$, and $L$ be given by

$$
\varepsilon:=\frac{h_{0}}{\left|\log h_{0}\right|}, \quad r_{1}:=\varepsilon^{1+\gamma / 2}, \quad \frac{1}{(1+\gamma / 2)^{L}}<\frac{1}{2} .
$$

Since $v(x) \leq \phi_{0}(x)$ for all $x \in \Omega$, and

$$
0 \leq \phi_{0}\left(x_{0}\right)=\rho\left(r_{1}\right)-r_{1} \rho^{\prime}\left(r_{1}\right)<\left|\frac{\log \varepsilon}{\log r_{1}}\right|^{L}=\frac{1}{(1+\gamma / 2)^{L}}<\frac{1}{2},
$$

we infer that (3.4) is valid.

4. It remains to verify (3.6) for $\phi_{0}$. Since

$$
\phi_{0}(x)=\phi_{0}\left(x_{0}\right)+\frac{\rho^{\prime}\left(r_{1}\right)}{K} a(x), \quad \rho^{\prime}\left(r_{1}\right) \leq C \frac{\left\lfloor\left.\log h_{0}\right|^{\gamma / 2}\right.}{h_{0}^{1+\gamma / 2}},
$$

invoking (3.8) we obtain

$$
\left|\Im\left(\phi_{0} ; x_{0}\right)-\phi_{0}\left(x_{0}\right)\right| \leq C h_{0}^{\gamma / 2}\left|\log h_{0}\right|^{\gamma / 2} .
$$

This shows that (3.6) holds with an exponent $<\gamma / 2$. Finally, applying Theorem 4 we conclude the proof.

Corollaries 4 and 5 yield in particular Theorem 1 of the introduction.

The above two impossibility results pertain to the topic of almost reproducing affine functions. We now turn our attention to the issue of almost reproducing piecewise linear functions, or equivalently to quasi-invariance of $S_{1}(\Omega ; h)$. We note that the Chen-Nochetto operator $\mathfrak{I}$ does not approximate the canonical basis functions $\phi_{i}$ with any order, namely,

$$
\left|1-\Im\left(\phi_{i} ; x_{i}\right)\right| \geq C>0 .
$$

Our last result in this section demonstrates that it is impossible to improve upon this. 
Corollary 6. Let $S(\Omega ; h)$ be a finite element space. For $1 \leq p \leq N$ there is no linear, bounded, and positive operator $\mathfrak{I}: W_{p}^{1}(\Omega) \rightarrow S(\Omega ; h)$ (resp. $\mathfrak{I}: \stackrel{\circ}{W}_{p}^{1}(\Omega) \rightarrow$ $S(\Omega ; h)$ ) which satisfies (3.5), i.e., is first order accurate in $L_{p}(\Omega)$, along with the local pointwise error estimate for some $\gamma>0$

$$
\left|1-\mathfrak{I}\left(1_{0} ; x_{i}\right)\right|+\left|1-\mathfrak{I}\left(\phi_{i} ; x_{i}\right)\right| \leq C h_{i}^{\gamma} .
$$

Here $\phi_{i}$ is the $i$-th canonical basis function of $S_{1}(\Omega ; h)$ for any node $x_{i}$ in $\bar{\Omega}$ and $1_{0}$ is 1 (resp. $x_{i}$ is such that $\operatorname{supp}\left(\phi_{i}\right)$ does not intersect $\partial \Omega$ and $1_{0}$ is the function defined in (2.4) ).

Proof. We consider first the case $W_{p}^{1}(\Omega)$. Let $v \in W_{p}^{1}(\Omega)$ be either the auxiliary function of Corollary 4, for $p<N$, or of Corollary 5 for $p=N$; thus (3.3) holds. Let $x_{0}:=x_{i}$ and the affine function $a(x)$ be replaced by the piecewise affine function $\tilde{a}(x):=h_{i}\left(1-\phi_{i}(x)\right)$. Note that

$$
\tilde{a}(x) \geq K\left|x-x_{0}\right|, \quad \forall x \in N_{0},
$$

where $N_{0}$ is the star around $x_{0}$. The barrier function is now given by $\phi_{0}(x):=$ $\varphi\left(\frac{\tilde{a}(x)}{K}\right)$ with $\varphi$ as in the proof of Corollaries 4 and 5 , respectively. This time $\phi_{0}(x)$ is constant outside $N_{0}$, but so is $v(x)$, and the domination in (3.4) still holds. We then argue as before to check (3.6).

On the other hand, when dealing with $\stackrel{\circ}{W}_{p}^{1}(\Omega)$ we simply multiply both $v$ and $\phi_{0}$ by the piecewise linear function $1_{0}$ of (2.4). Since $\operatorname{supp}\left(\phi_{i}\right)$ is interior to $\Omega$, we deduce that $v 1_{0}$ is arbitrarily close to $1_{0}$ in $\mathscr{W}_{p}^{1}(\Omega)$, and $\phi_{0} 1_{0} \in \stackrel{\circ}{W}_{p}^{1}(\Omega)$ is a barrier function satisfying (3.4) and (3.6). We finally apply Theorem 4 in conjunction with Remark 3.2 to complete the proof.

Remark 3.3. In view of Remark 3.1, (3.11) can be weakened, for $1 \leq p<N$, to

$$
\left|1-\mathfrak{I}\left(1_{0} ; x_{i}\right)\right|+\left|1-\mathfrak{I}\left(\phi_{i} ; x_{i}\right)\right|=o(1) .
$$

Remark 3.4. Consider the operator $\mathfrak{I}: \stackrel{\circ}{W}_{p}^{1}(\Omega) \rightarrow \stackrel{\circ}{S}_{1}(\Omega ; h)$ given by

$$
\mathfrak{I}(u ; x):=\left(\frac{2}{h} \int_{0}^{h} u\right) \phi_{1}(x)+\sum_{i=2}^{I-1}\left(\frac{1}{2 h} \int_{x_{i-1}}^{x_{i+1}} u\right) \phi_{i}(x)+\left(\frac{2}{h} \int_{x_{I}}^{1} u\right) \phi_{I}(x),
$$

where we are using the notation of Remark 2.1. We see that $\mathfrak{I}\left(\phi_{1} ; x_{1}\right)=\mathfrak{I}\left(\phi_{I} ; x_{I}\right)=$ 1 and thus (3.11) is valid at $x=x_{1}, x_{I}$. This construction extends to $N \geq 2$ and $N-1<p \leq N$ as follows: if $x_{i}$ is a node connected to a boundary node $x_{j}$ through an element edge $\gamma_{i j}$, we set $\mathfrak{I}\left(u ; x_{i}\right)=\frac{2}{\left|\gamma_{i j}\right|} \int_{\gamma_{i j}} u$. We deduce that the assumption on the support of $\phi_{i}$ is necessary in Corollary 6 .

\section{Restriction to SECOND ORDER ACCURACY}

In this section we investigate Korovkin's classical result in the context of piecewise polynomial approximation on finite element spaces $S(\Omega ; h)$ for general partitions $\mathfrak{T}$ of $\Omega$. We prove, following Korovkin [7, that a linear, bounded, and positive finite element operator $\mathfrak{I}$ cannot be more than second order accurate in $L_{1}$ provided some additional, but natural, conditions on $S(\Omega ; h)$ and $\mathfrak{I}$ hold.

We consider the $h$-method; that is, we assume that there is an integer $k \geq 1$ independent of $\mathfrak{T}$ such that all shape functions of $S(\Omega ; h)$ are piecewise polynomials 
of degree $\leq k$ : for all $u \in S(\Omega ; h)$,

$$
\left.u\right|_{T} \in \Pi_{k}(T), \quad \forall T \in \mathfrak{T} .
$$

We further assume that the interpolation operator $\mathfrak{I}$ is local: there is a function $\rho(h)$ such that $\rho(h) \downarrow 0$ as $h=h(x) \downarrow 0$ and

$$
\mathfrak{I}(u ; x) \quad \text { depends only on the values of } u \text { in } B(x, \rho(h)) \cap \bar{\Omega},
$$

where $B(x, \rho)$ is the ball of radius $\rho$ centered at $x$. As is easily seen from (1.7), $\rho(h)=C_{4} h$ for the Chen-Nochetto operator.

Let $\chi_{0} \in C_{0}^{\infty}(\Omega)$ be a nonnegative cut-off function which is 1 in a neighborhood of $\Omega_{0}$, where $\Omega_{0} \Subset \Omega$ is a fixed region in $\Omega$. Then for any function $f \in L_{1}(\Omega)$ we may write $\mathfrak{I}(f ; x)$ to mean $\mathfrak{I}\left(\chi_{0} f ; x\right)$ which, in light of (4.2), is defined only in terms of $f$ provided $x \in \Omega_{0}$ and the mesh size $h_{\mathfrak{T}}:=\|h\|_{L_{\infty}(\Omega)}$ is sufficiently small. We also assume that

$$
\left\|\mathfrak{I}\left(\chi_{0}\right)\right\|_{L_{\infty}\left(\Omega_{0}\right)} \leq C
$$

with $C$ independent of $\mathfrak{T}$. We then have the following result.

Theorem 5. Let $\mathfrak{T}$ be a general edge-to-edge partition of $\Omega$, and let $\mathfrak{I}: \stackrel{\circ}{C}(\Omega) \rightarrow$ $\stackrel{\circ}{S}(\Omega ; h)$ or $\mathfrak{I}: C(\bar{\Omega}) \rightarrow S(\Omega ; h)$ be a linear, bounded, and positive operator which satisfies (4.1)-(4.3). Then, for $h_{\mathfrak{T}}$ sufficiently small, we have

$$
\begin{aligned}
C \operatorname{meas}\left(\Omega_{0}\right) \leq & \left\|h^{-2}\left(|x|^{2}-\mathfrak{I}\left(|y|^{2} ; x\right)\right)\right\|_{L_{1}\left(\Omega_{0}\right)} \\
& +\sum_{n=1}^{N}\left\|h^{-2}\left(x_{n}-\mathfrak{I}\left(y_{n} ; x\right)\right)\right\|_{L_{1}\left(\Omega_{0}\right)} \\
& +\left\|h^{-2}(1-\mathfrak{I}(1 ; x))\right\|_{L_{1}\left(\Omega_{0}\right)} .
\end{aligned}
$$

Remark 4.1. A positive operator $\mathfrak{I}$ must thus fail to be more than second order accurate already on one of the local polynomial functions $|x|^{2}=\sum_{n=1}^{N} x_{n}^{2}, x_{n}$ $(1 \leq n \leq N)$, or 1 (even with the error measured in $L_{1}$, the weakest $L_{p}$ space).

Remark 4.2. Theorem[5] does not demand any regularity of the meshes, not even a maximum angle condition.

Theorem 5 relies on a classical result of Korovkin for polynomial approximation, Lemma 4.3 below [7 Theorem 17, p.128 and p.132]. For the reader's convenience, we give a proof following Lorentz [8, Theorem 3, p.94], for which we need two preliminary results. A positive operator satisfies

$$
|\mathfrak{I}(f ; x)| \leq \mathfrak{I}(|f| ; x), \quad \forall x \in \Omega
$$

together with the Cauchy-Schwarz inequality

$$
|\mathfrak{I}(f g ; x)|^{2} \leq \mathfrak{I}\left(f^{2} ; x\right) \mathfrak{I}\left(g^{2} ; x\right), \quad \forall x \in \Omega .
$$

The inequality (4.5) results from applying $\mathfrak{I}$ to $|f| \pm f \geq 0$, and (4.6) is a consequence of the following nonnegative quadratic expression in $\lambda \in \mathbb{R}$ :

$$
0 \leq \mathfrak{I}\left((f+\lambda g)^{2} ; x\right)=\mathfrak{I}\left(f^{2} ; x\right)+2 \lambda \mathfrak{I}(f g ; x)+\lambda^{2} \mathfrak{I}\left(g^{2} ; x\right) .
$$

For notational convenience we introduce the following error functions:

$$
e_{0}(x)=\mathfrak{I}(1 ; x)-1, \quad e_{1, n}(x)=\mathfrak{I}\left(y_{n} ; x\right)-x_{n}, \quad e_{2}(x)=\mathfrak{I}\left(|y|^{2} ; x\right)-|x|^{2},
$$

where $1 \leq n \leq N$. Korovkin's result is now the following. 
Lemma 4.3. Let $\mathfrak{I}$ be a positive linear operator, and let $t \in \Omega$ be arbitrary. Then

$$
\begin{aligned}
\mid \Im(|y-t| ; x)- & |x-t||\leq| x-t|| e_{0}(x) \mid \\
& +\Im(1 ; x)^{1 / 2}\left(e_{2}(x)-2 \sum_{n=1}^{N} x_{n} e_{1, n}(x)+|x|^{2} e_{0}(x)\right)^{1 / 2} .
\end{aligned}
$$

Proof. We have, by the triangle inequality,

$$
|| y-t|-| x-t|| \leq|y-x| \text {. }
$$

Since $|x-t|$ acts as a constant for $\mathfrak{I}$, we can write

$$
\mathfrak{I}(|y-t| ; x)=\mathfrak{I}(|y-t|-|x-t| ; x)+|x-t| \mathfrak{I}(1 ; x),
$$

whence, using that $\mathfrak{I}$ is positive together with (4.5), we get

$$
|\mathfrak{I}(|y-t| ; x)-| x-t|| \leq|x-t||\mathfrak{I}(1 ; x)-1|+\mathfrak{I}(|y-x| ; x) .
$$

In view of (4.6), we then obtain

$$
\begin{aligned}
& \mathfrak{I}^{2}(|y-x| ; x) \leq \Im(1 ; x) \mathfrak{I}\left(|y-x|^{2} ; x\right) \\
& \quad=\Im(1 ; x)\left(\Im\left(|y|^{2}-2 y \cdot x+|x|^{2} ; x\right)-|x|^{2}+2 x \cdot x-|x|^{2}\right) \\
& \quad=\mathfrak{I}(1 ; x)\left(\left(\mathfrak{I}\left(|y|^{2} ; x\right)-|x|^{2}\right)-2 \sum_{n=1}^{N} x_{n}\left(\mathfrak{I}\left(y_{n} ; x\right)-x_{n}\right)+|x|^{2}(\mathfrak{I}(1 ; x)-1)\right) .
\end{aligned}
$$

This proves the assertion.

The following three lemmas will enable us to prove Theorem 5 without any shape regularity assumption on the mesh $\mathfrak{T}$.

Lemma 4.4. For any simplex $T$ in $\mathbb{R}^{N}$ let $P$ be a parallelipiped with a vertex at a vertex $V$ of $T$ and edges given by $1 / N$ times the edges of $T$ meeting at $V$. Then $P \subset T$.

Proof. This is clear using barycentric coordinates.

Lemma 4.5. Let $\Pi_{k}(-d, d)$ be the set of polynomials of degree $\leq k$ on the interval $(-d, d)$. There exists a constant $C$, depending only on $k$ and $N$, such that

$$
\min _{\varphi \in \Pi_{k}(-d, d)} \int_{-d}^{d}|| r|-\varphi(r)|^{2} r^{N-1} d r \geq C d^{2+N} .
$$

Proof. Scaling to $(-1,1)$, with $\varphi(r) \in \Pi_{k}(-d, d)$ corresponding to $\phi(\rho)=d^{-1} \varphi(d \rho) \in$ $\Pi_{k}(-1,1)$, we obtain

$$
\int_{-d}^{d}|| r|-\varphi(r)|^{2}|r|^{N-1} d r=d^{2+N} \int_{-1}^{1}|| \rho|-\phi(\rho)|^{2}|\rho|^{N-1} d \rho .
$$

Since $\Pi_{k}(-1,1)$ is finite dimensional and $|\rho| \notin \Pi_{k}(-1,1)$, the assertion follows.

Lemma 4.6. Let $T$ and $P$ be as in Lemma 4.4 with one edge of $P$ on one of the longest edges of $T$, and let $t$ be the center of gravity of $P$. There exists a constant $C>0$, solely depending on $k$ and $N$, such that

$$
\min _{\varphi \in \Pi_{k}(T)}|||\cdot-t|-\varphi \|_{L_{2}(T)} \geq C h(t) \operatorname{meas}(T)^{1 / 2} .
$$


Proof. We may assume for ease of notation that $t=0$. One edge of $P$ is on one of the longest edges of $T$, of size $h(t)$; we let $S_{1}$ and $S_{2}$ be two parallel faces of $P$ separated by $h(t) / N$. Let $P_{1}$ and $P_{2}$ denote the two pyramids formed by $S_{1}$ and $t$, and $S_{2}$ and $t$, respectively. Then meas $\left(P_{1} \cup P_{2}\right)$ is a fixed fraction of meas $(T)$, depending only on $N$, namely,

$$
\operatorname{meas}\left(P_{1} \cup P_{2}\right)=\frac{N !}{2 N^{N}} \operatorname{meas}(T) .
$$

Introducing polar coordinates on $P_{1} \cup P_{2}$, we can write

$$
\int_{P_{1} \cup P_{2}}|| x|-\varphi(x)|^{2} d x=\int_{\Theta} \int_{-d(\theta)}^{d(\theta)}|| r|-\varphi(r, \theta)|^{2} r^{N-1} d r d \omega(\theta),
$$

where the integration in $\theta$ extends over the appropriate set of polar angles $\Theta$. Since by construction $d(\theta)$ is comparable to $h(t)$, Lemma 4.5 gives

$$
\begin{aligned}
\int_{P_{1} \cup P_{2}}|| x|-\varphi(x)|^{2} d x & \geq C h^{2+N} \int_{\Theta} d \omega(\theta) \\
& \geq C h^{2} \int_{\Theta} \int_{-d(\theta)}^{d(\theta)} r^{N-1} d r d \omega(\theta)=C h^{2} \operatorname{meas}(T) .
\end{aligned}
$$

This proves the lemma.

Proof of Theorem 5 . We recall that $\chi_{0}$ is a cut-off function which is 1 in a neighborhood of $\Omega_{0}$, and that we write $\Im(f ; x)$ for $x \in \Omega_{0}$ to mean $\Im\left(\chi_{0} f ; x\right)$. Since $\mathfrak{I}$ is a local operator, that is, $\mathfrak{I}$ satisfies (4.2), we infer that the definitions of $\mathfrak{I}\left(|y|^{2} ; x\right), \mathfrak{I}\left(y_{n} ; x\right)$ and $\mathfrak{I}(1 ; x)$ are unaffected by the choice of $\chi_{0}$ if $x \in \Omega_{0}$ for $h_{\mathfrak{T}}$ sufficiently small. We thus conclude that (4.7) is valid provided both $x, t \in \Omega_{0}$. Let $T$ be a simplex in $\mathfrak{T}$ contained in $\Omega_{0}$, and let $t$ be as in Lemma 4.6. Since $\Omega$ is bounded, using Lemmas 4.6 and 4.3 , we see that

$$
\begin{aligned}
& C h(t)^{2} \operatorname{meas}(T) \leq \int_{T}\left(|x-t|\left|e_{0}(x)\right|\right. \\
& \left.\quad+\mathfrak{I}^{1 / 2}\left(\chi_{0} ; x\right)\left(\left|e_{2}(x)\right|+2 \sum_{n=1}^{N}\left|e_{1, n}(x) \| x_{n}\right|+|x|^{2}\left|e_{0}(x)\right|\right)^{1 / 2}\right)^{2} \\
& \leq C\left(h^{2}(t)\left\|e_{0}^{2}\right\|_{L_{1}(T)}+\left\|e_{2}\right\|_{L_{1}(T)}+\sum_{n=1}^{N}\left\|e_{1, n}\right\|_{L_{1}(T)}+\left\|e_{0}\right\|_{L_{1}(T)}\right) \\
& \leq C\left(\left\|e_{2}\right\|_{L_{1}(T)}+\sum_{n=1}^{N}\left\|e_{1, n}\right\|_{L_{1}(T)}+\left\|e_{0}\right\|_{L_{1}(T)}\right),
\end{aligned}
$$

where we also used (4.3) in the last two steps. Equivalently, we have

$$
C \operatorname{meas}(T) \leq C\left(\left\|h^{-2} e_{2}\right\|_{L_{1}(T)}+\sum_{n=1}^{N}\left\|h^{-2} e_{1, n}\right\|_{L_{1}(T)}+\left\|h^{-2} e_{0}\right\|_{L_{1}(T)}\right) .
$$

Let $\tilde{\Omega}_{0}$ be the union of all simplices of $\mathfrak{T}$ contained in $\Omega_{0}$. Then

$$
C \operatorname{meas}\left(\tilde{\Omega}_{0}\right) \leq\left(\left\|h^{-2} e_{2}\right\|_{L_{1}\left(\Omega_{0}\right)}+\sum_{n=1}^{N}\left\|h^{-2} e_{1, n}\right\|_{L_{1}\left(\Omega_{0}\right)}+\left\|h^{-2} e_{0}\right\|_{L_{1}\left(\Omega_{0}\right)}\right) .
$$

For $h_{\mathfrak{T}}$ sufficiently small, meas $\left(\tilde{\Omega}_{0}\right)$ is comparable with meas $\left(\Omega_{0}\right)$, which gives the desired lower bound. The proof of Theorem 5 is thus complete. 
Remark 4.7. Even though it does not pay to enlarge the range of $\mathfrak{I}$ beyond $S_{1}(\Omega ; h)$ to increase the rate of convergence, one may wonder about positive interpolation operators $\mathfrak{I}$ with larger range. As a simple example, consider the following construction for $N=1$ : let $S_{2}(\Omega ; h)$ be the space of continuous piecewise quadratics over $\mathfrak{T}$, and, on the master interval $(0,1)$, set

$$
\mathfrak{I}(u ; x)=u(0)(1-x)+u(1) x+(2 u(1 / 2)-(u(0)+u(1))) x(1-x) .
$$

The range of $\mathfrak{I}$ is all the piecewise quadratics, $\mathfrak{I}$ reproduces the piecewise linears, and $\mathfrak{I}$ is positive since

$$
\Im(u ; x)=u(0)(1-x)^{2}+u(1) x^{2}+2 u(1 / 2) x(1-x) .
$$

Obviously, such an operator cannot also preserve quadratics locally as this would violate Theorem 5. In fact, $x^{2}$ is not preserved in this example.

Acknowledgments. The present work started while the authors were enjoying the hospitality of the Mittag-Leffler Institute in 1998. They thank Douglas Arnold for an interesting question leading to Theorem 1, They also thank the referees for suggesting improvements in the presentation. R. H. Nochetto was partially supported by NSF Grants DMS-9623394 and DMS-9971450. L. B. Wahlbin was partially supported by NSF Grant DMS-9703683.

\section{REFERENCES}

[1] S.C. Brenner and L.R. Scott, The Mathematical Theory of Finite Element Methods, SpringerVerlag, 1994. MR 95f:65001

[2] F. Brezzi and M. Fortin, Mixed and Hybrid Finite Element Methods, Springer-Verlag, 1991. MR 92d:65187

[3] Z. Chen and R.H. Nochetto, Residual type a posteriori error estimates for elliptic obstacle problems, Numer. Math. 84 (2000), 527-548. MR 2001c:65134

[4] P.G. Ciarlet, Basic Error Estimates for Elliptic Problems, in Handbook of Numerical Analysis, Vol II (Finite Element Methods (Part 1)), P.G. Ciarlet and J.L. Lions eds, North-Holland, 1991, 17-351. CMP 91:14

[5] Ph. Clément, Approximation by finite element functions using local regularization, RAIRO Anal. Numer. 9 (1975), 77-84. MR 53:4569

[6] S. Hilbert, A mollifier useful for approximations in Sobolev spaces and some applications to approximating solutions of differential equations, Math. Comp. 27 (1973), 81-89. MR 48:10047

[7] P.P. Korovkin, Linear Operators and Approximation Theory, Hindustan Publishing Company, Delhi, 1960. MR 27:561

[8] G.G. Lorentz, Approximation of Functions, Holt, Rinehart and Winston, New York, 1966. MR 35:4642

[9] R. H. Nochetto, K. Siebert, and A. Veeser, Pointwise a posteriori error control of elliptic obstacle problems (to appear).

[10] L.R. Scott and S. Zhang, Finite element interpolation of nonsmooth functions satisfying boundary conditions, Math. Comp. 54 (1990), 483-493. MR 90j:65021

[11] G. Strang, Approximation in the finite element method, Numer. Math. 19 (1972), 81-98. MR 46:4677

[12] R. Verfürth, A Review of A Posteriori Error Estimation and Adaptive Mesh-Refinement Techniques, Wiley-Teubner, 1996.

Department of Mathematics and Institute for Physical Science and Technology, University of Maryland, College Park, Maryland 20742

E-mail address: rhn@math.umd.edu

Department of Mathematics, Cornell University, Ithaca, New York 14853

E-mail address: wahlbin@math.cornell.edu 